\title{
Infección por Dipylidium caninum en un preescolar. Presentación del caso y revisión de la literatura
}

\author{
Patricia Neira O., Leonor Jofré M. y Nelson Muñoz S.
}

\section{Dipylidium caninum infection in a 2 year old infant: case report and literature review}

Dipylidiasis is a zoonotic parasitic infection caused by the dog tapeworm Dipylidium caninum; it affects both feline and canine species and accidentally, humans. In Chile, as well as in other countries, it is an uncommon infection. A case of a 2 year old child from Casablanca, (a city located in the Valparaíso Region), with an infection by D. caninum, is presented. Clinical manifestations are reviewed, as well as epidemiology in domestic and wild animals, cases among the published national literature and its treatment and prevention strategies.

Key words: Dipylidiasis, Dipylidium caninum, dog tapeworm, zoonoses.

Palabras clave: Dipilidiasis, dipilidiosis, Dipylidium caninum, tenia del perro, zoonosis.

\section{Introducción}

$\mathrm{L}$ a dipilidiasis es una zoonosis parasitaria producida por Dipylidium caninum, gusano aplanado en sentido dorsoventral, de la familia Dilepidiidae, orden Cyclophyllidea, Subclase Eucestoda. Es un cestodo común en cánidos y félidos domésticos y silvestres, quienes son sus hospederos definitivos.

El hombre lo adquiere accidentalmente al ingerir a los hospederos intermediarios infectados, la pulga del perro (Ctenocephalides canis), la pulga del gato (Ctenocephalides felis) y ocasionalmente por la pulga del hombre (Pulex irritans) o el piojo del perro (Trichodectes canis) ${ }^{1}$.

La infección en el hombre se denomina dipilidiosis, la mayoría de los casos se presentan en lactantes y preescolares, lo que sugiere una mayor exposición a los hospederos intermedios, por el estrecho contacto con las mascotas, que pueden lamer la cara del niño, sus juguetes y utensilios de alimentación. En adultos, en cambio, es poco frecuente $\mathrm{e}^{2,3}$.

Presentamos el caso clínico de un preescolar con dipilidiosis y su posterior discusión.

\section{Caso Clínico}

Preescolar de 2 años 8 meses de edad, sexo masculino, sano, sin antecedentes mórbidos de importancia, con controles ocasionales con el pediatra en el último año. Estado nutricional eutrófico según $\mathrm{P} / \mathrm{E}$ y $\mathrm{P} / \mathrm{T}$. En el examen físico destacaba el desaseo personal.

La familia procedía de la zona rural de Casablanca, Región de Valparaíso, donde se dedicaban a la crianza de vacunos y aves. La casa con piso de madera, disponía de agua potable y alcantarillado, patio de tierra. Tenían tres perros, uno de ellos adulto y un gato de tres años. Las mascotas no contaban con control veterinario. La madre del niño refería la presencia de abundantes pulgas. El niño jugaba frecuentemente con sus mascotas.

El paciente fue derivado a la Cátedra de Parasitología para control post tratamiento de una Hymenolepis diminuta, diagnosticada en otro centro, donde había consultado por dolor abdominal.

Al interrogar dirigidamente a la madre destacaba el antecedente de la eliminación de elementos blanquecinos móviles por las deposiciones. Se realizó un estudio copro-parasitológico y test de Graham, que fueron negativos y se solicitó una muestra de los elementos eliminados por las deposiciones del niño (Figura 1).

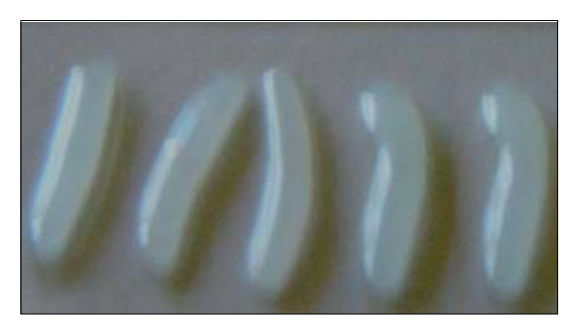

Figura 1. Proglótidas de $D$. caninum, eliminadas por el paciente.

\author{
Universidad de Valparaíso, \\ Chile \\ Facultad de Medicina \\ Escuela de Medicina \\ Departamento de Preclínicas. \\ Cátedra de Parasitología (PNO, \\ NMS) \\ Instituto de Salud Pública de \\ Chile \\ Laboratorio de Microbiología Clínica \\ (UM). \\ Financiado, parcialmente, por \\ Dirección de Investigación de la \\ Universidad de Valparaíso (DIPUV \\ 01/2005)
}

Recibido: 25 septiembre 2007 Aceptado: 11 septiembre 2008

Correspondencia a: Patricia Neira Otero patricia.neira@uv.cl 
Figura 2. Huevos de Dipylidium caninum contenidos en la membrana ovígera.
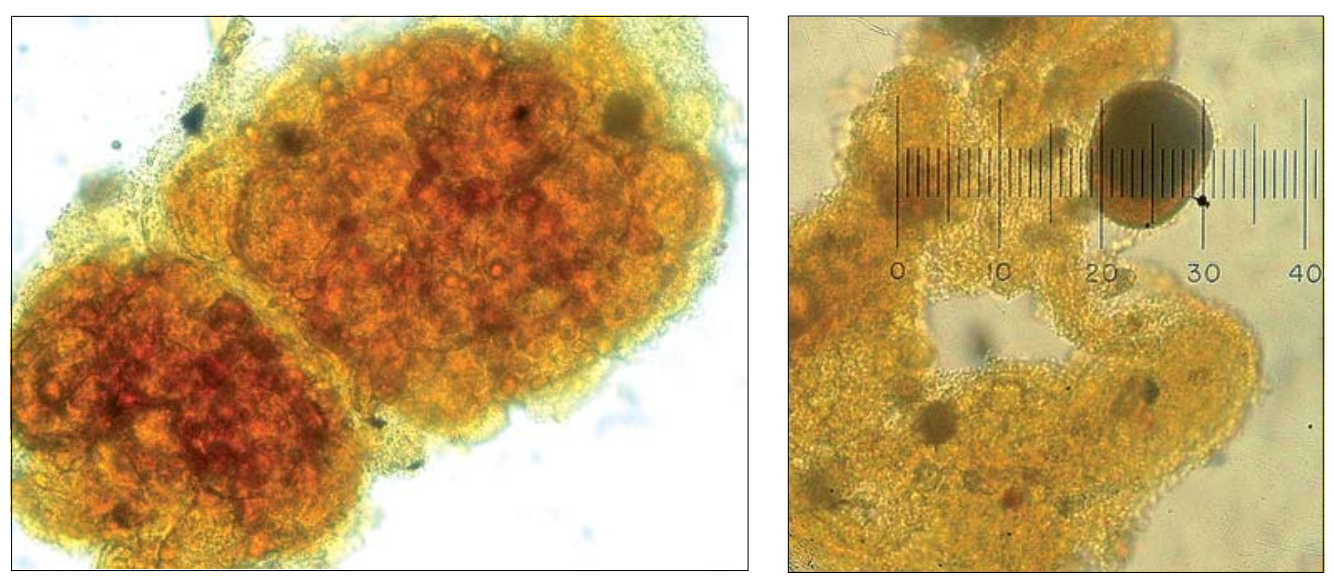

Figura 3. Huevo de Dipylidium caninum libre.

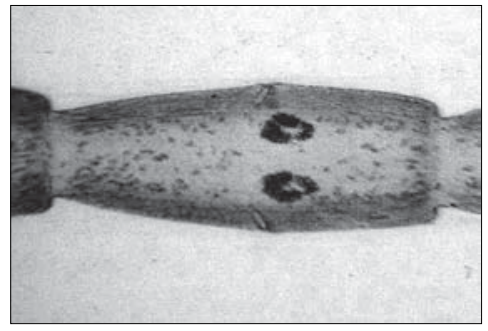

Figura 4. Proglótida de doble poro de Dipylidium caninum.

La observación macro y microscópica de estos elementos permitió la visualización de un doble poro genital característico y la presencia de huevos agrupados en una membrana o cápsula ovígera, con liberación de huevos (Figuras 2-4). De acuerdo a los hallazgos macro y microscópico se hizo el diagnóstico de dipilidiosis.

El paciente recibió medidas preventivas para dipilidiosis y tratamiento con praziquantel por una vez. Acudió a control con estudio copro-parasitológico de deposiciones post tratamiento, a los 3 y 6 meses después, que fueron todos negativos. No volvió a eliminar proglótidas y se encuentra actualmente asintomático. En el último control se informó de la muerte del perro adulto.

\section{Discusión}

Dipylidiun caninum fue descrito por primera vez el año 1715 por Dubois. Es una zoonosis de distribución mundial, la infección se ha reportado en todos los continentes a excepción de la Antártida.

Epidemiología. La frecuencia de infección por $D$. caninum en cánidos y félidos domésticos capturados en Chile el siglo pasado, era de $39,9 \%{ }^{4}$. Estudios realizados posteriormente en las ciudades de Valdivia, Nuble y Santiago encontraron una prevalencia entre 11,4 y $54,2 \%{ }^{5-8}$.

El año 2006 se encontró en 2,1 y 2,2\% de las muestras fecales estudiadas en perros de Santiago ${ }^{9,10}$. En la ciudad de Valparaíso se detectó en 1,6\% de los perros estudiados (Oschilewski D., datos no publicados).

En países como México se ha encontrado hasta en $60 \%$ de muestras de autopsias de perros y en $52 \%$ de muestras de perros sanos ${ }^{11,12}$. En España, en 13,2\% de los perros sin hogar ${ }^{13,14}$ y en Brasil $0,7 \%{ }^{15}$.

La parasitación es mayor en perros mayores de un año (Oschilewski D., datos no publicados) $)^{8,10}$, con cantidades variables de tenias, de 1 a 426 ejemplares/ animal $^{15-19}$. En Chile, la máxima carga parasitaria comunicada ha sido de 140 cestodos ${ }^{17}$.

En gatos se ha encontrado D. caninum en 45 a $51 \%$ de los animales sacrificados, con 1 a 300 ejemplares por animal ${ }^{20-21}$. En Santiago, en gatos que presentaban síntomas digestivos, se encontró en 6,9\% de las muestras estudiadas 9 . En Valparaíso se ha encontrado en 2/ 15 cachorros (Neira P., datos no publicados). La presencia del cestodo es mayor en cachorros de gatos ${ }^{9}$.

En animales silvestres como el zorro se ha encontrado en $2,6 \%$ en Italia $^{22}, 3,8 \%$ en Inglaterra ${ }^{23}, 3,1$ y $50 \%$ en $\mathrm{Grecia}^{24}, 19,4 \%$ en Jordania ${ }^{25}, 0,9 \%$ en Bélgica ${ }^{25}, 2 \%$ en la República Eslovaca ${ }^{26,27}$ y $5,5 \%$ en España ${ }^{28}$. La eventualidad de transmisión de agentes etiológicos de zoonosis a partir de cánidos y félidos silvestres al hombre es remota; sin embargo, actúan como reservorios de agentes patógenos comunes al perro, con el cual el hombre tiene mayor posibilidad de contacto ${ }^{29}$.

En animales en cautiverio como zorros, aguará guazú, jaguar, gato montés y gato moro, $D$. caninum tiene una incidencia de $13,7 \% \%^{30}$. En un estudio efectuado en animales del zoológico de Quilpué, Región de Valpa- 
raíso, no se detectó su presencia en cánidos ni félidos (Neira P., datos no publicados).

El tratamiento de esta parasitosis en animales de compañía se realiza con praziquantel. Se trata, a contar de los cuatro meses de vida, en el perro y seis meses en el gato, por las reacciones de hipersensibilidad secundarias a la liberación de los antígenos, ocasionado por la muerte de los parásitos. El tratamiento debe repetirse cada tres meses. Para el control de pulgas se indica un collar antipulgas.

Biología. Dipylidium caninum es un cestodo de color blanquecino, puede alcanzar una longitud promedio de $30 \mathrm{~cm}(10-70 \mathrm{~cm})$. El escólex es romboidal de 350 a $400 \mu$, posee cuatro ventosas, un rostelo apical, cónico y retráctil, capaz de evaginarse llegando a una longitud de $185 \mu$ o invaginarse totalmente dentro del escólex (Figuras 5 y 6). Tiene un promedio de 4 a 6 coronas de ganchos, que pueden variar de 1 a 8 , dependiendo de la edad del parásito. Estos ganchos fijan al cestodo en la pared del intestino delgado.

Las proglótidas semejan una cadena de "granos de arroz o pepitas de melón". Presentan dos juegos de órganos reproductores localizados en extremos opuestos, lo que las hace fácilmente diferenciables de otras tenias (Figura 6). Se liberan grávidas al ambiente, ya sea por su propia motilidad al franquear el esfínter anal, que es lo habitual, o bien, junto a las deposiciones, que es excepcional. En el ambiente externo se desintegran y liberan 50 o más paquetes de huevos, que se forman después de la reproducción sexual, pueden quedar aislados o, lo más frecuente, dentro de una delgada membrana, llamada cápsula ovígera.

Los huevos miden entre 20 y $40 \mu$ de diámetro. Si el huevo es ingerido por un hospedero intermediario (pulga), eclosiona la oncósfera (embrión hexacanto), que penetra la pared intestinal, invade el hemocele del insecto (cavidad del cuerpo), y se convierte en procercoide y posteriormente en una larva cisticercoide llamada Cryptocystis trichodectis ${ }^{31}$.

La larva cisticercoide tiene un período de incubación de 10 a 25 días, alcanza su madurez mientras el insecto cumple con su propia metamorfosis (holometábola). Reside en la cavidad del cuerpo de los insectos y la transmisión al hospedero definitivo ocurre por la ingestión de los insectos infectados con larvas cisticercoides $^{32}$.

El imago alberga el estado larval infectante (cisticercoide) para los hospederos definitivos. El cisticercoide se desarrolla, en aproximadamente un mes, a la forma adulta (tenia) en el intestino delgado, donde se adhiere mediante su escólex. Debido a que una pulga puede contener múltiples larvas de la tenia, es posible la infección con más de un ejemplar.

Clínica en animales de compañia. Los hospederos definitivos son el perro y el gato y en ellos no ocasiona síntomas, a excepción de prurito anal. Sólo cuando la carga parasitaria es alta puede provocar trastornos gastrointestinales, que incluso pueden llevar a una obstrucción intestinal. Las manifestaciones clínicas varían, dependiendo entre otros factores, de la edad, sexo, raza y condición física de los animales. Un signo característico es la observación de las proglótidas en la zona perineal de los animales o en los lugares donde descansan ${ }^{33-34}$.

Manifestaciones clínicas en humanos. La mayoría de los casos de dipilidiosis ocurre en niños, un tercio de ellos en lactantes bajo 6 meses de edad. Muchas veces pasa inadvertida o no se diagnostica, porque no se visualizan las proglótidas o los pacientes no refie-
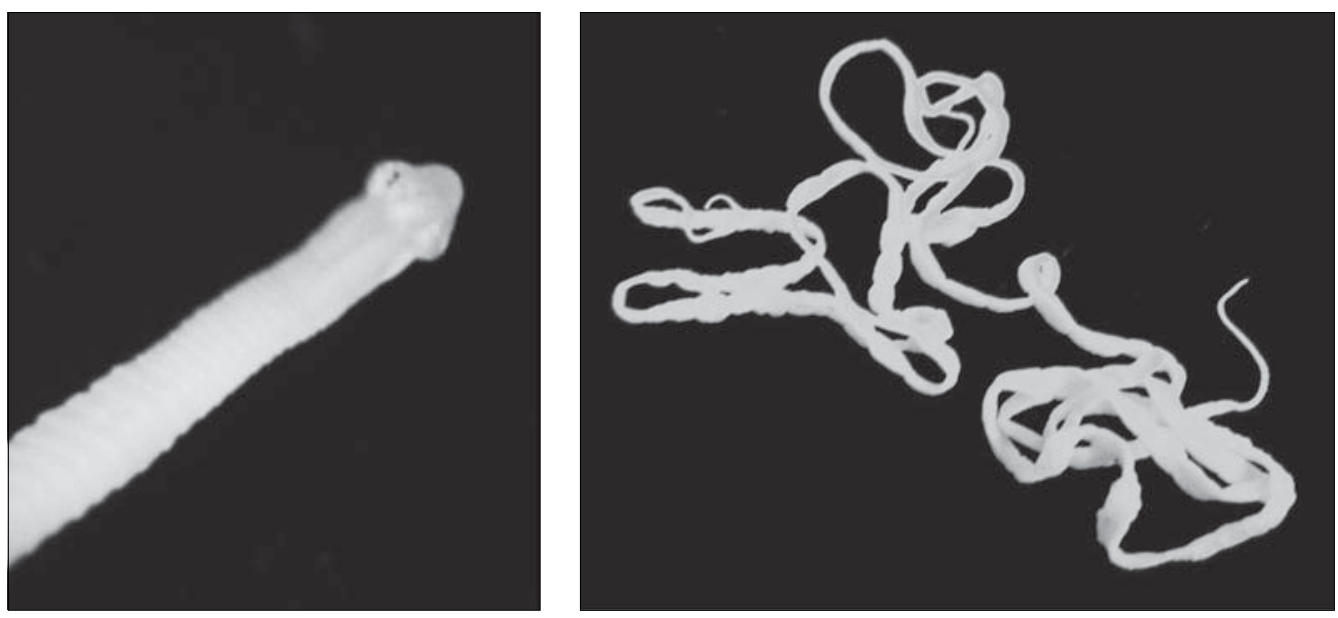

Figuras 5 y 6. Extremo anterior con rostelo invaginado y ejemplar de Dipylidium caninum. 
ren síntomas. La presencia de esta zoonosis, se asocia a malas condiciones higiénicas y contacto estrecho con mascotas. Los niños se infectan cuando son lamidos por un perro o gato con pulgas infectadas, las que pueden ser ingeridas accidentalmente o ser tragadas cuando se encuentra en pisos o patios ${ }^{33}$.

La infección tiene un período pre-patente de 20 días. La carga parasitaria se relaciona directamente con el número de larvas cisticercoides presentes en las pulgas y por la cantidad de insectos ingeridos. La carga parasitaria en humanos generalmente es baja, con escasos reportes de infección múltiple ${ }^{35,36}$. El cestodo no se multiplica en el hombre, porque no es su hospedero definitivo.

La mayoría de las veces la infección es asintomática. Cuando presenta síntomas, las manifestaciones son vagas e inespecíficas e incluyen diarrea, inquietud, agitación en lactantes, dolor epigástrico, constipación, palpitaciones cardíacas. En niños mayores ocasiona prurito y dolor anal. Los síntomas ceden con la expulsión del o los ejemplares de la tenia ${ }^{37-39}$. Se puede asociar a irritabilidad, insomnio, distensión abdominal, dolor abdominal, cólico, meteorismo, pérdida de apetito, baja de peso y reacción alérgica ${ }^{37}$. Los niños pueden eliminar proglótidas en deposiciones y es posible de encontrar en los pañales. En ocasiones puede encontrarse eosinofilia en el hemograma ${ }^{39}$. Tiende a confundirse con Enterobius vermicularis ${ }^{40-41}$. La patogenicidad en el hombre se relaciona a la absorción de metabolitos del parásito ${ }^{38}$.

En nuestro país, en un estudio realizado en 51.020 personas se detectó $D$. caninum en 18 de ellos $(0,7 \%)^{42}$. Existen publicados en la literatura médica nacional más

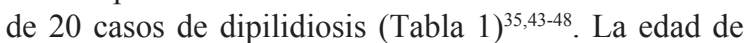
los casos publicados varía entre 2 meses y 4 años, 15/ 17 casos eran lactantes, nueve hombres y 8 mujeres.

\begin{tabular}{|c|c|c|c|c|c|}
\hline Año y procedencia & Edad & Síntomas & Sexo & Diagnóstico & Tratamiento \\
\hline 1952-Stgo $0^{35}$ & $5 \mathrm{~m}$ & Asintomático & M & Proglótida obtenida de pañal & Metoquina. Eliminó 6 ejemplares \\
\hline 1952-Stgo 43 & $5 \mathrm{~m}$ & Asintomático & M & $\begin{array}{l}\text { Eliminación de proglótidas } \\
\text { móviles }\end{array}$ & Metoquina. Eliminó 6 ejemplares \\
\hline $1952-\operatorname{Stg}^{43}$ & $5 \mathrm{~m}$ & Asintomático & M & - & Acranyl \\
\hline 1959-1963 Stgo 44 & 2 a & $\begin{array}{l}\text { Diarrea, cólico, irritabilidad, insomnio, } \\
\text { distensión abdominal }\end{array}$ & M & Eliminación de proglótidas & Acranyl. Eliminó 1 ejemplar \\
\hline $1959-1963 \mathrm{Stgo}^{44}$ & $17 \mathrm{~m}$ & $\begin{array}{l}\text { Diarrea, cólico, irritabilidad, insomnio, } \\
\text { distensión abdominal }\end{array}$ & $\mathrm{F}$ & Eliminación de proglótidas & Metoquina. Eliminó 1 ejemplar \\
\hline $1959-1963 \mathrm{Stgo}^{44}$ & $4 \mathrm{~m}$ & Asintomático & $\mathrm{F}$ & Eliminación de proglótidas & Niclosamida. Eliminó 1 ejemplar \\
\hline $1959-1963 \mathrm{Stgo}^{44}$ & $4 \mathrm{~m}$ & $\begin{array}{l}\text { Irritabilidad, insomnio, distensión } \\
\text { abdominal }\end{array}$ & $\mathrm{F}$ & Eliminación de proglótidas & Niclosamida. Eliminó 1 ejemplar \\
\hline 1959-1963 Stgo 44 & $2 \mathrm{~m}$ & Diarrea y cólicos & M & Eliminación de proglótidas & Niclosamida. Eliminó 1 ejemplar \\
\hline $1959-1963$ Stgo $^{44}$ & $7 \mathrm{~m}$ & $\begin{array}{l}\text { Diarrea, cólico, irritabilidad, insomnio, } \\
\text { distensión abdominal }\end{array}$ & $\mathrm{F}$ & Eliminación de proglótida & Niclosamida. Eliminó 1 ejemplar \\
\hline $1959-1963 \mathrm{Stgo}^{44}$ & $15 \mathrm{~m}$ & Distensión abdominal & $\mathrm{F}$ & Eliminación de proglótida & Niclosamida. Eliminó 1 ejemplar \\
\hline $1959-1963 \mathrm{Stgo}^{44}$ & $2 \mathrm{~m}$ & $\begin{array}{l}\text { Diarrea, cólico, irritabilidad, insomnio, } \\
\text { distensión abdominal }\end{array}$ & $\mathrm{F}$ & Eliminación de proglótidas & Niclosamida. Eliminó 1 ejemplar \\
\hline $1959-1963 \mathrm{Stgo}^{44}$ & $13 \mathrm{~m}$ & $\begin{array}{l}\text { Irritabilidad, insomnio, distensión } \\
\text { abdominal }\end{array}$ & $\mathrm{F}$ & Eliminación de proglótidas & Niclosamida. Eliminó 1 ejemplar \\
\hline 1962 Concepción ${ }^{45}$ & $4 a$ & Cólico intestinal, diarrea y meteorismo & M & Eliminación de proglótidas & $\begin{array}{l}\mathrm{N}-\left(2^{\prime} \text {-cloro-4'-nitrofenil) }\right. \\
\text { 5-clorosalicilamida }\end{array}$ \\
\hline 1963 Concepción ${ }^{45}$ & $4 \mathrm{~m}$ & -- & M & Autopsia: D. caninum en íleon & - \\
\hline 1966 Santiago $^{46}$ & $18 \mathrm{~m}$ & Baja de peso, decaimiento, irritabilidad. & M & Eliminación de proglótidas & Niclosamida \\
\hline \multirow[t]{2}{*}{1969 Rancagua $^{47}$} & $15 \mathrm{~m}$ & Intranquilidad, Insomnio & M & Eliminación de proglótidas & Niclosamida \\
\hline & & & & (junto con las deposiciones) & \\
\hline $1987 \mathrm{Stgo}^{48}$ & $14 \mathrm{~m}$ & - & $\mathrm{F}$ & $\begin{array}{l}\text { Eliminación de proglótidas } \\
\text { móviles }\end{array}$ & Praziquantel una dosis \\
\hline
\end{tabular}


Presentaron diarrea, cólicos y distensión abdominal 11 de ellos, asintomáticos 4. Se visualizó las proglótidas en 15, un caso fue un hallazgo de autopsia. El tratamiento usado en la mayoría de los casos fue niclosamida.

Diagnóstico. El diagnóstico de certeza de esta parasitosis se logra al identificar las proglótidas, que semejan un grano de arroz y las cápsulas ovígeras; generalmente no se observan huevos libres en deposiciones.

Las proglótidas son de color blanquecino, se diferencian de otras tenias por su tamaño, características morfológicas y la observación microscópica, sin tinción entre dos portaobjetos, de los dos poros genitales (Figura 7). El personal de laboratorio debe estar familiarizado con las características antes señaladas, para evitar confusión con otras especies de tenias, o larvas de moscas.

Dada la infrecuencia de esta infección en humanos, las infecciones pueden no ser reconocidas o subdiagnosticadas, principalmente porque las proglótidas en las deposiciones pueden pasar inadvertidas.

Tratamiento. Se realiza con praziquantel, un antihelmíntico de amplio espectro. Actúa aumentando la permeabilidad de la membrana celular en los gusanos susceptibles, produce pérdida del calcio intracelular, contracciones masivas y parálisis de la musculatura. Esto es seguido por adherencia de fagocitos a los parásitos y muerte. El medicamento actúa desintegrando la tenia en el intestino, motivo por el que no se observa en deposiciones. Es bien tolerado, con pocos efectos adversos. No se ha demostrado teratogenicidad, pero no se recomienda su uso en mujeres embarazadas ni en nodrizas, porque se excreta por la leche materna. Es bien absorbido en el tracto gastrointestinal. Está disponible en comprimidos de 150 y $500 \mathrm{mg}^{49,50}$.

La niclosamida fue usada en el tratamiento de la dipilidiosis. Inhibe la fosforilación oxidativa mitocondrial del parásito. Necesita de una preparación intestinal previa del paciente, con régimen líquido durante la tarde y noche anterior. Al día siguiente se administra el comprimido en ayunas, que debe ser masticado e ingerido con una taza de té ${ }^{47}$ (Tabla 2).

Prevención. Las medidas de prevención recomendadas en esta parasitosis son:

- Evitar que los niños jueguen con animales que tienen pulgas, control de ellas, tanto en ambientes interiores como al aire libre.

- Inspeccionar posible infección en animales domésticos.

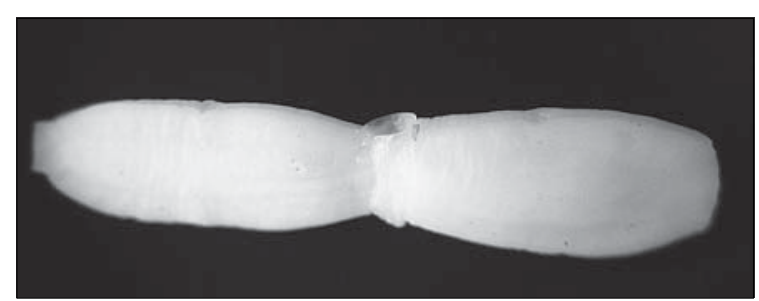

Figura 7. Proglótidas de Dipylidium caninum.

Tabla 2. Dosificación de anticestocidas

\begin{tabular}{lll} 
Fármaco & Niclosamida & Praziquantel \\
$\begin{array}{l}\text { Presentación y nombre } \\
\text { comercial }\end{array}$ & Yomesan® comp $500 \mathrm{mg}$ & $\begin{array}{l}\text { Cesol® comp } 150 \mathrm{mg} \\
\text { Cisticid }{ }^{\circledR} \text { comp } 500 \mathrm{mg}\end{array}$ \\
Administración & Oral & Oral \\
& Dosis única & Dosis única \\
Observaciones & Requiere preparación intestinal & $\begin{array}{l}\text { Fármaco de elección } \\
\text { No requiere preparación }\end{array}$ \\
& No absorbible & Venta bajo receta médica \\
& Actualmente no está & \\
& disponible en el país & No usar en mujeres \\
& & $\begin{array}{l}\text { embarazadas ni nodrizas } \\
\text { Se administra con líquidos }\end{array}$ \\
& & 0 comidas \\
Dosis en adultos & $2 \mathrm{~g}$ & $400 \mathrm{mg} / \mathrm{día}$ \\
\hline Niños $>4$ años & $1,5 \mathrm{~g}$ & $10-20 \mathrm{mg} / \mathrm{kg}$ de peso \\
Niños $<4$ años & $1 \mathrm{~g}$ & $10-20 \mathrm{mg} / \mathrm{kg}$ de peso \\
\hline
\end{tabular}

- Control veterinario de las mascotas.

- Retiro de patios y áreas recreacionales públicas de los excrementos de perros.

- Enterrar o bien embolsar y eliminar en la basura.

- Evitar que los niños besen a las mascotas o sean lamidos por ellas.

\section{Conclusiones}

Dipylidium caninum es una zoonosis que raramente causa infección en el hombre. Se asocia al contacto estrecho con mascotas e ingestión de pulgas infectadas con el cisticercoide, que son sus hospederos intermediarios. Los niños son los más afectados, especialmente los lactantes. El diagnóstico se sospecha visualizando las proglótidas en deposiciones, región perianal o en los pañales. El tratamiento se realiza con praziquantel. Entre las medidas de prevención recomendadas está el control de pulgas de las mascotas con collares antipulgas, mantener un control veterina- 
rio, desparasitación periódica y evitar que los niños besen o sean lamidos por sus mascotas.

\section{Resumen}

La dipilidiasis es una zoonosis parasitaria provocada por Dipylidium caninum, teniasis que afecta a cánidos, félidos $\mathrm{y}$, en forma accidental, al hombre. En
Chile, al igual que en otros países, es una infección poco frecuente. Se presenta el caso de un preescolar con una infección por $D$. caninum, residente en la comuna de Casablanca, Región de Valparaíso. Se revisan las manifestaciones producidas por este agente, la epidemiología en animales domésticos y salvajes, los casos publicados en la literatura nacional, así como su tratamiento y las medidas de prevención.

\section{Referencias}

1.- Bartsocas C S, Von Graevenitz A, Blodgett F. Dipylidium infection in a 6 month old infant. J Pediatr 1966; 69: 814-4.

2.- Jackson D, Crozier W J, Andersen S E J, Giles W, Bowen TE. Dipylidiasis in a 57 year-old woman. Med J Austral 1977; 2: $740-1$.

3.- Casasbuenas P. Infección por Dipydilium caninum. Rev Col Gastroenterol 2005; 20: 86-8

4.- Neghme A, Rivera G, Álvarez M. Algunas zoonosis parasitarias en perros vagos de la ciudad de Santiago. Bol Chile Parasitol 1955; 10: $73-5$.

5.- Torres P, Ramos M, Carrasco L, Neumann M, Franjola R, Navarrete N, et al. Protozoos, helmintos y artrópodos parásitos del perro doméstico en la ciudad de Valdivia, Chile. Bol Chile Parasitol 1974; 29: 18-23.

6.- Oberg C, Franjola R, Leían V. Helmintos del perro doméstico (Canis familiaris) en la ciudad de Valdivia, Chile. Bol Chile Parasitol 1979; 34: 21-6.

7.- Rubilar L, Zapata L, Moreno G, Cerda S. Prevalencia de Echinococcus granulosus y de otros cestodos del perro en la comuna de El Carmen, Nuble. Parasitol al Día 1985; 9: 55-7.

8.- Alcaíno H, Tagle I. Estudio sobre enteroparasitosis del perro en Santiago. Bol Chile Parasitol 1970; 25: 5-8.

9.- López J, Abarca K, Paredes P, Inzunza E. Parásitos intestinales en caninos y felinos con cuadros digestivos en Santiago, Chile: Consideraciones en Salud Pública. Rev Méd Chile 2006; 134: 193-200.

10.- Gorman T, Soto A, Alcaíno H. Parasitismo gastrointestinal en perros de comunas de Santiago de diferente nivel socioeconómico. Parasitol Latinoam 2006; 61: 126-32.

11.- Eguía-Aguilar P, Cruz-Reyes A, Martínez Maya J. Ecological analysis and description of the intestinal helminths present in dogs in Mexico City. Vet Parasitol 2005; 127: 139-46.

12.- Rodríguez-Vivas R, Bolio-González M, Domínguez-Alpizar J, Aguilar-Flores J,
Cob-Galera L. Prevalencia de Dipylidium caninum en perros callejeros de la ciudad de Mérida, Yucatán, México. Rev Biomed 1996; 7: 205-10.

13.- Martínez-Carrasco C, Berriatúa E, Garijo M, Martínez J, Alfonso F D, de Ybañez R R. Epidemiological study of non-systemic parasitism in dogs in southeast Mediterranean Spain assessed by coprological and post-mortem examination. Zoonoses Public Health 2007; 54: 195-203.

14.- Martínez-Moreno $\mathrm{F} J$, Hernández S, López-Cobos E, Becerra C, Acosta I, Martínez-Moreno A. Estimation of canine intestinal parasites in Córdoba (Spain) and their risk to public health. Vet Parasitol 2007; 143:7-13.

15.- Oliveira-Sequeira T, Amarante A, Ferrari T, Nunes L. Prevalence of intestinal parasites in dogs from Sao Paulo State, Brazil. Vet Parasitol 2002; 103: 19-27.

16.- Soulsby E J. Parasitología y enfermedades parasitarias en los animales domésticos. $7^{\mathrm{a}}$ Ed. México: Iinteramericana, 1987. Págs 102-23.

17.- Oberg C, Franjola R, Leían V. Helmintos del perro doméstico (Canis familiaris) en la ciudad de Valdivia, Chile. Bol Chile Parasitol 1979; 34: 21-6.

18.- Le-Riche P D, Soe A K, Alemzada Q, Sharifi L. Parasites of dogs in Kabul, Afganistan. Br Vet J 1988; 144: 370-3.

19.- Jenkins D, Andrew P. Intestinal parasites in dogs from an aboriginal community in New South Wales. Australian Vet J 1993; 3:1156.

20.- Torres P, Hott A, Boehmeald H. Protozoos, helmintos y artrópodos en gatos de la ciudad de Valdivia y su importancia para el hombre. Arch Med Vet 1972; 4: 20-9.

21.- Alcaíno H, Gorman T, Larenas I. Fauna endoparasitaria del gato doméstico en una zona urbana marginal de la Región Metropolitana de Chile. Parasitol al Día 1992; 16: $139-42$

22.- Guberti V, Poglayen G. Parasitic zoonoses: Survey in foxes (Vulpes vulpes) in the northen appenines. Hystrix 1991; 3: 167-73.
23.- Richards D T, Harris S, Lewis J W. Epidemiological studies on intestinal helminth parasites of rural and urban red foxes (Vulpes vulpes) in the United Kingdom. Vet Parasitol, 1995; 59: 39-51.

24.- Papadopoulos H, Himonas C, Papazahariadou M, Antoniadu-Soritiadou K. Helminths of foxes and other wild carnivores from rural areas in Greece. J Helminthology 1997; 71: 227-31.

25.- El-Shehabi F S, Abdel-Afez S K, Kamhawi S A. Prevalence of intestinal helminths of dogs and foxes from Jordan. Parasitol Res 1999; 85: 928-34.

26.- Vervaeke M, Dorny P, De Bruyn L, Vercammen F, Jordanes K, Van Den Berge Koen, et al. A survey of intestinal helminths of red foxes (Vulpes vulpes) in northern Belgium. Acta Parasitol 2005; 50: 221-7.

27.- Letková, V, Lazar P, Urlík J, Goldová M, Kocišová $\mathrm{A}$, Košuthová $\mathrm{L}$, et al. The red fox (Vulpes vulpes L.) as a source of zoonoses. Vet Archiv 2006; 76: S73-S81.

28.- Urquhart G M, Armour J, Duncan J L, Dunn A M, Jennings Y F. Veterinary Parasitology 1987. Longman Scientific and Technical. Essex. 286pp.

29.- Segovia J, Torres J, Llaneza M, Feliu Y C. Helminths in the wolf, Canis lupus, from the north-western Spain. J. Helmintol 2001; 75: $183-92$.

30.- Martínez F, Binda J, Maza Y. Determinación de platelmintos por coprología en carnívoros silvestres. Universidad Nacional del Nordeste. Comunicaciones Científicas y Tecnológicas 2005.

31.- Soulsby E J. Parasitología y enfermedades parasitarias en los animales domésticos. 7a Ed. México: Interamericana, 1987: págs102123.

32.- Weisse M E, Mullins J K, Moffett K S. A neonate with worms. Clin Infect Dis 2008; 46: 1745, 1786-8.

33.- Bowman D D. Georgis' Parasitology for Veterinarians. 6a Ed. Philadelphia: Saunders Company, 1995, Págs 145-6.

34.- Molina C P, Ogburn J, Adegboyega P. Infection by Dipylidium caninum in an infant. Arch Pathol Lab Med 2003; 127: e157-9. 
35.- Fanta E. Parasitismo humano por Dipylidium caninum (Linneo, 1758) (Resumen de un caso). Bol Inf Paras Chilenas 1952; 7: 29.

36.- Reis C J, Perry F M, Evans N. Dipylidium caninum in an infant. Eur J Pediatr 1992; 151: 502-3.

37.- Wong M H. Multiple infestations with Dipylidium caninum in an infant. Can Med Assoc J 1955; 72: 453-6.

38.- Chappell C L, Enos J P, Penn H. Dipylidium caninum and under recognized infections in infant and children. Pediatr Infect Dis J. 1990; 9: 745-6.

39. - Neafie R C, Marty A M. Unusual infections in humans. Clin Microbiol Rev 1993; 6: 3456.

40. - Samkari A, Kiska D L, Riddell S W, Wilson K, Weiner L B, et al. Dipylidium caninum mimicking recurrent Enterobius vermicularis (pinworm) Infection. Clin Pediatr (Phila) 2008; 28: 397-8

41.- Hamrick H J, Drake W R, Jones HM, Preston Askew A, Weatherly N F. Two cases of Dipylidium caninum (dog tapeworm) infection in children: update on an old problem. Pediatrics 1983; 72: 114-7.

42.- Reyes H, Doren G, Inzunza E. Teniasis humana. Frecuencia actual de la infección por diferentes especies en Santiago de Chile. Bol Chil Parasitol 1972; 27: 23-9.

43. - Fanta E. Parasitismo humano por Dipylidium caninum (Linneo, 1758) Comunicación de dos casos. Rev Chil Pediat 1952; 23: 393-6.

44.- Belmar R. Dipylidium caninum en niños. Comunicación de 13 casos y tratamiento con un derivado de la salicilamida. Bol Chil Parasit 1963; 18: 63-7.
45.- Bull F, Oyarce R. Dos casos de dipilidiasis humana en Concepción. Bol Chil Parasit 1966; 21: 22.

46.- Link A, Cassorla E. Dipylidium caninum en un lactante. Rev Chile Pediatr 1966; 37 : 33-4.

47.- Nicolet G. Teniasis por Dipylidium caninum en un niño. Bol Chil Parasit 1969; 24: 150-1.

48.- Schenone H, Thompson L, Quero M S. Infección por Dipylidium caninum en una niña pequeña tratada con prazicuantel. Bol Chil Parasitol 1987; 42: 74-5.

49.- Atías A. Tratamiento de las parasitosis y tablas terapéuticas. Parasitología Médica, Edit Mediterráneo, Stgo de Chile 1998. Capítulo 66, págs 579-99.

50.- Craig P, Ito A. Intestinal cestodes. Curr Opin Infect Dis 2007; 20: 524-32. 